\title{
Inkomplette Querschnittlähmung nach Vertebroplastie
}

\author{
Peter Wendsche
}

Die 64-jährige weibliche Patientin nach Hausunfall (Fall vom Stuhl) wurde zu uns am 6. Tag nach dem Unfall von einem auswärtigen Versorgungskrankenhaus mit der Diagnose Kompressionsfraktur L1 (Typ A1.2) verlegt (Abb.1). Es bestanden zunächst keine neurologischen Ausfälle im Verlegungsbericht. Eine präoperative neurologische Kontrolluntersuchung ergab allerdings ein inkomplettes Konussyndrom, was als periradikuläres Dekompensationssyndrom gewertet wurde.

Am 11. Tag nach dem Unfall wurde in Allgemeinnarkose eine modifizierte Vertebroplastie (Kavoplastie) durchgeführt. Dabei werden unter Bildwandlerkontrolle in üblicher Weise die Pedikel des zu instrumentierenden Wirbels aufgesucht. Über Führungsdrähte werden in beide Pedikel Perforatoren eingeführt (Abb. 2). Über die Perforatoren werden die im Durchmesser $7 \mathrm{~mm}$ starken Applikationskanülen eingeführt (Abb.3). Mit einem Spezialinstrument, was sich nach Einführen abwinkeln lässt (Abb. 4), wird im Wirbelkörper durch Drehen eine Höhle geschaffen, in die später der Biozement mit niedrigem Druck eingefüllt wird (Abb.5). Während der Operationsprozedur wurde kein Zementaustritt beobachtet (E-Flow Zement).

Während der Aufwachphase bestand eine zunächst komplette Querschnittlähmung. Der Patientin wurde sofort eine Solumedrol ${ }^{\circledR}$-Infusionstherapie verordnet in der üblichen Dosierung (30 mg in $100 \mathrm{ml}$ physiologischer Lösung als Bolus, danach in einer Dosierung von $5,4 \mathrm{mg} / \mathrm{kg} / \mathrm{h})$. Es wurde sofort eine CT durchgeführt ( $2 \mathrm{~h}$ nach Beendigung der Operation), in der Zementaustritt in den dorsalen Abschnitten des Wirbelkanals von Th10 bis L2 $(11 \mathrm{~cm})$ nachgewiesen

OP-JOURNAL 2008; 24: 192-193

(c) Georg Thieme Verlag KG Stuttgart • New York DOI 10.1055/s-2008-1039072

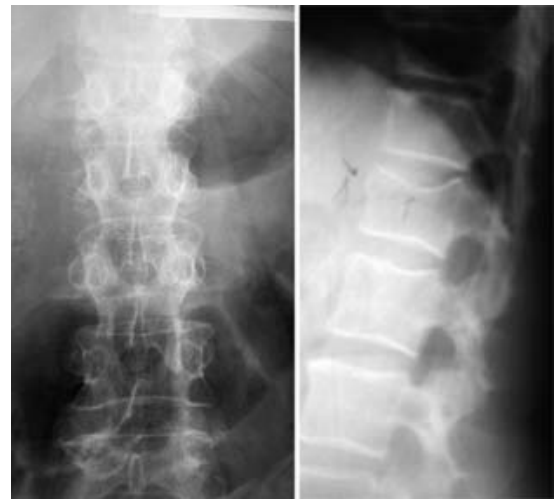

Abb. 1 Unfallbilder im a.-p. und seitlichen Strahlengang.

wurde (Abb. 6). Es wurde sofort eine Revisionsoperation veranlasst $(3,5 \mathrm{~h}$ nach Beendigung der Erstoperation). Dabei wurde erneut in Allgemeinnarkose eine Laminektomie im Ausmaße von Th9 bis L2 durchgeführt und der ausgetretene Zement en bloc entfernt. Danach wurde eine transpedikuläre Stabilisierung Th11-Th12-L2 mit einem winkelstabilen Implantat (USS) durchgeführt (Abb. 7).

Am darauffolgenden Tag wurde die Patientin von der Intensivstation auf die Querschnittgelähmtenstation verlegt, die sich im Hause befindet. Die Patientin wurde im üblichen Ausmaße der Rehabilitation für Querschnittgelähmte zugeführt, zunächst auf der schon erwähnten Querschnittgelähmtenstation im Hause (Postakutphase) und später in einem Rehabilitationszentrum für Querschnittgelähmte. Neben der üblichen Comprehensive Care wurde auch die IMF $^{\circledR}$-Therapie (Intention controlled Myofeedback) angewandt. An der Stabilität der operierten Wirbelsäule hat sich sowohl auf den Aufnahmen der 1-jährigen Kontrolle (Abb.8) als auch bei der letzten Nachuntersuchung im September 2008 (Abb.9) nichts verändert. Die auf der a.-p. Aufnahme zu sehende Skoliose ist klinisch nicht relevant. Da nach

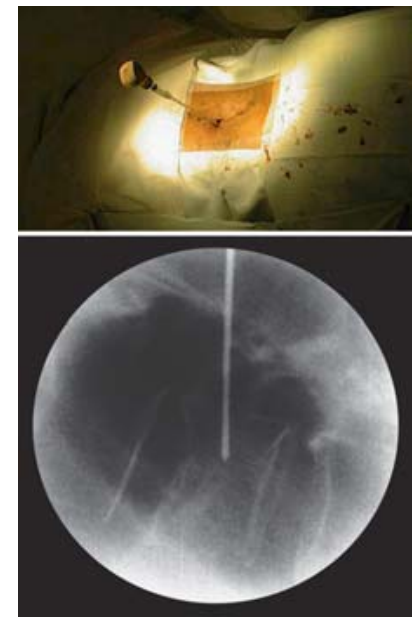

Abb. 2 Der Pedikelperforator ist in den Wirbelkörper transkutan eingeführt.

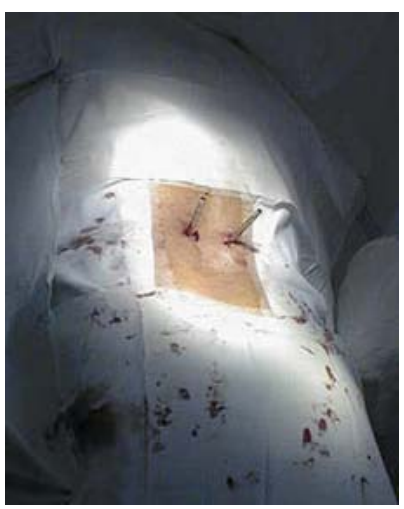

Abb. 3 Transkutan eingeführte Applikationskanülen.

der Revisionsoperation keine KontrollCT angefertigt wurde, ist der heutige Befund mit Nachweis von Restzement im hinteren Teil des Kanals (Abb.10) so gesehen auch für uns eine Überraschung.

Obwohl sich der neurologische Befund gebessert hat, braucht sie häusliche Hilfe. Mit fremder Hilfe steht sie relativ stabil (Abb.11), zum Gehen braucht sie einen niedrigen Abstützroller. Neurologisch hat sie eine spastische inkom- 


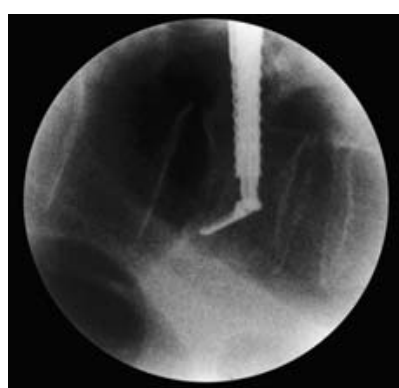

Abb. 4 Durch Drehen des Spezialinstruments wird im Wirbelkörper eine Höhle geschaffen.

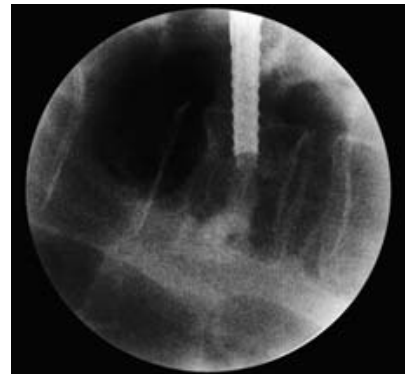

Abb. 5 Austritt des Biozements (E-Flow) aus der Applikationskanüle in den Wirbelkörper.

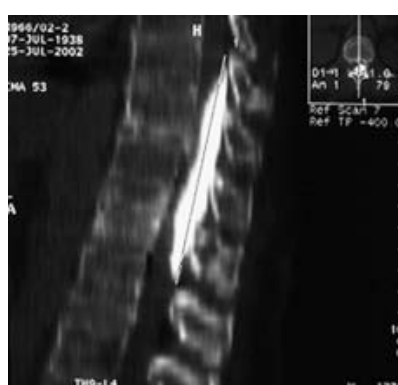

Abb. 6 Die postoperative sagittale CT-Rekonstruktion zeigt den ausgetretenen Zement im hinteren Bereich des Wirbelkanals.
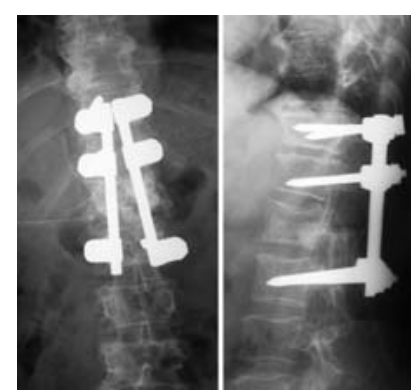

Abb. 7 Postoperative Röntgenaufnahmen nach der Revisionsoperation (Laminektomie) mit transpedikulärer Stabilisierung von Th11 über Th12 nach L1.

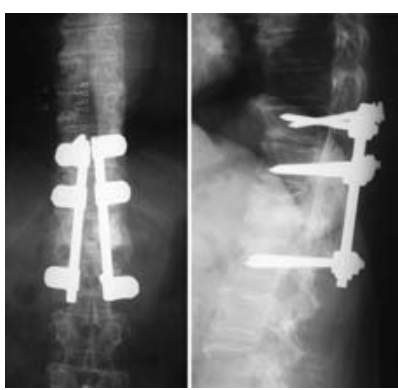

Abb. 8 Röntgenologische Kontrolle nach 1 Jahr. Kein Korrekturverlust.

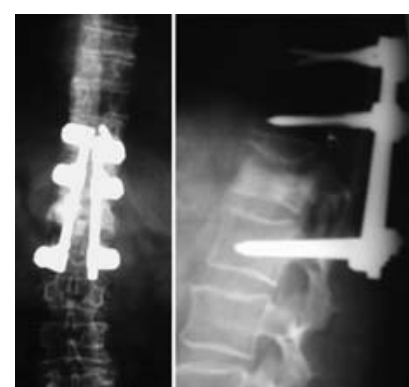

Abb.9 Röntgenologische Kontrolle nach 6 Jahren (max. Followup). Kein Korrekturverlust.

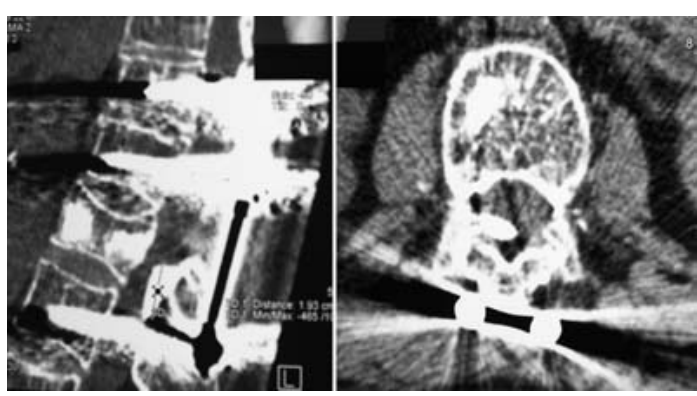

Abb. 10 Die CT-Aufnahmen (9/2008) zeigen den Restzement im Wirbelkanal.

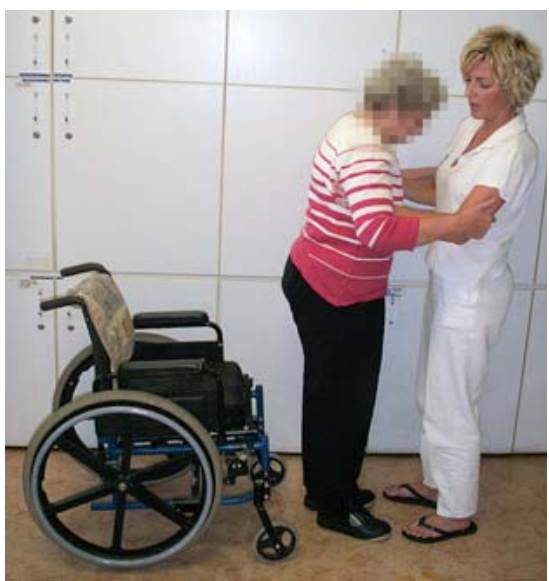

Abb. 11 Die Patientin braucht 6 Jahre nach der Operation Gehhilfe.

plette Lähmung mit Hyperanästhesien in der linken unteren Extremität und mit Störung beider Sensitivitätsqualitäten (Störung sowohl des lateralen als auch hinteren Rückenmarkstranges). Neurophysiologisch ist die Störung als Bild einer Myelopathie zu werten.

\section{Fazit}

- Im Gegensatz zur Vertebroplastie bei Haemangiomen ist die Methode bei Kompressions-/Berstungsbrüchen mit einem Risiko des Zementaustritts belastet.

- Die Instrumentarien für Vertebroplastie mit dünnen Applikationskanülen sind dem beschriebenen System vorzuziehen.

- Grundsätzlich sollte bei Kompressions-/Berstungsbrüchen präoperativ eine $\mathrm{CT}$ angefertigt werden, die die knöchernen Verhältnisse der Hinterkante und der Pedikel beschreibt.

- Im beschriebenen Falle ist es mit großer Wahrscheinlichkeit zum retrograden Zementaustritt über den instrumentierten Pedikel gekommen. Ob die Pedikelfraktur primär vorlag, oder durch die Applikation der 7-mmKanüle entstanden ist, kann nicht exakt evaluiert werden.

\section{Prof. Dr. med. Peter Wendsche} Vorstand

Unfallchirurgische Klinik mit Abteilung für Querschnittgelähmte Úrazová nemocnice Brno

Masaryk Universität

Ponavka 6

66250 Brno

Tschechien

E-Mail: p.wendsche@unbr.cz 